\title{
ON GALOIS PROJECTIVE GROUP RINGS
}

\section{GEORGE SZETO}

Mathematics Department

Bradley University

Peoria, Illinois 61625 U.S.A. and

\section{LINJUN MA}

Mathematics Department Zhongshen University

Guangzhou, P.R. China

(Received September 19, 1989 and in revised form December 10, 1989)

ABSTRACT. Let $A$ be a ring with $1, C$ the center of $A$ and $G$ ' an inner automorphism group of $A$ induced by $\left\{U_{\alpha}\right.$ in $A / \alpha$ in a finite group $G$ whose order is invertible\}. Let $A^{\prime}$ be the fixed subring of $A$ under the action of G'. If $A$ is a Galcis extension of $A^{G}$ with Galois group $G^{\prime}$ and $C$ is the center of the subring $\sum_{\alpha} A^{\prime} U_{\alpha}$ then $A=\sum_{\alpha} A^{G^{\prime}} U_{\alpha}$ and the center of $A^{G^{\prime}}$ is also $C$. Moreover, if $\Sigma_{\alpha} A^{G '} U_{\alpha}$ is Azumaya over $C$, then $A$ is a projective group ring.

KEY WORDS AND PHRASES. Central Galois extensions, projective Eroup rings, Azumaya algebras. 1980 AMS SUBJECT CLASSIFICATION CODE. 16A74, 16A16, 13 BO2.

\section{INTRODUCTION.}

Galois extensions for rings and Azumaya algebras have been intensively investigated (see References). F.R. DeMeyer ([1]) characterizes a central Galois algebra with an inner Galois group in terms of an Azumaya projective group algebra. That is, if $A$ is a central Galois algebra over $C$ with an inner Galois group $G^{\prime}$ induced by the units $\left\{U_{\alpha}\right.$ $/ \alpha$ in a finite group $G\}$, then $A$ is a projective group algebra $C_{f}$ where $f$ is a factor set $f(\alpha, \beta)=U_{\alpha} U_{\beta} U_{\alpha \beta}^{-1}$ for $\alpha, \beta$ in $G$. Conversely, if $C_{f}$ is Azumaya over $C$, then it is Galois over $C$ with an inner Galois group G' induced by $\left\{U_{\alpha}\right\}$ ([1], Theorems 2 and 3 ). In the present paper, we shall study a Galois extension A over a ring $A^{\prime}$ (not necessarily its center $C)$. It will be shown that if $A$ is Galois with Galois group $G^{\prime}$ and if the subring $\sum_{\alpha} A^{\prime} U_{\alpha}$ has center $C$, ther $A=$ $\sum_{\alpha} A^{G^{\prime}} U_{\alpha}$ such that the center of $A^{G^{\prime}}$ is also $C$. In addition, if $A^{G^{\prime}}$ is separable over $C$, then $A$ becomes a projective group ring over $A^{G '}$, $A=A^{G} G_{f}$ (that is, the coefficient ring $C$ in a projective group C-algebra is replaced by a ring $A^{G '}$ as defined in [1]). In this case, $A$ is an Azumaya C-algebra. Conversely, if $\sum_{\alpha} A^{G} U_{\alpha}$ is an C-Azumaya C-algebra such that $\left\{U_{\alpha}\right\}$ are free over $C$, then $A=A^{G} G_{f}$ and is Galois over $A^{G}$ such that $A^{G^{\prime}}$ has center $C$. Our results generalize the characterization for a central Galois algebra as given by F.R. DeMeyer ([1], Theorems 
2 and 3). This paper was done under the support from the Advanced Research Institute of Zhongshan University, P.R. China, and revised under the suggestions of the referee. We would like to thank the referee for his valuable comments to relate our paper to the work of $T$. Kanzaki.

2. BASIC DEFINITIONS.

Throughout, we assume that $A$ is a ring with 1 , $C$ the center of A, G' the inner automorphism Eroup of $A$ induced by the set of units $\left\{U_{\alpha} / \alpha\right.$ in a finite group $G$ whose order is invertible $\}$, and $A^{G^{\prime}}=$ $\left\{a\right.$ in $A / \alpha^{\prime}(a)=a=U_{\alpha} a U_{\alpha}^{-1}$ for each $\alpha^{\prime}$ in $\left.G^{\prime}\right\}$. The projective group ring $R G_{f}$ over a ring $R$ is a ring with an $R$-basis $\left\{U_{\alpha} / \alpha\right.$ in G\} such that $U_{\alpha} U_{\beta}=U_{\sigma} f(\alpha, \beta)$ where $\alpha \beta=\sigma$ in $G$ and $r U_{\alpha}=\Psi_{\alpha} r$ for each $r$ in $R, \alpha, \beta$ in $G$ and $f(\alpha, \beta)=U_{\alpha} U_{\beta} U_{\alpha \beta}^{-1}$. A separable extension $T$ over $S$ is a ring extension $T$ over its subring $S$ such that there exist elements $\left\{a_{i}, b_{i}\right.$ in $T, i=1, \ldots, m$ for some integer $m$ / $\sum a a_{i} b_{i}=\sum a_{i} b_{i} a$ for each $a$ in $T$ and $\left.\sum a_{i} b_{i}=1\right\}$ where is over S. Such a set $\left\{a_{i}, b_{i}\right\}$ is called a separable set for $T$. The separable S-algebra $T$ is a separable extension $T$ over $S$ which is contained in the center of $T$, and $T$ is an Azumaya $S$-algebra if $T$ is a separable algebra over its center $S$. $T$ is a Galois extension over $T^{G}$ with Galois group $G$ if $G$ is a finite automorphism group of $T$ and there exist elements $\left\{x_{i}, y_{i}\right.$ in $T, i=1, \ldots, k$ for some integer $k$ $/ \sum x_{i} y_{i}=1$ and $\sum x_{i} \alpha\left(y_{i}\right)=0$ for each $\alpha \neq 1$ in $\left.G\right\}$. Such a set $\left\{x_{j}, y_{i}\right\}$ is called a Galois set for $T$. A Galois extension $T$ over $T^{G}$ with Galois group $G$ is called a centralized Galois extension if $T^{G}$ has the same center as $T$.

3. GAICIS PROJECTIVE GROUP RINGS.

Throughout, we assume that $A$ is a ring with $1, C$ the center of $A$, G' an inner automorphism group of $A$ induced by $\left\{U_{\alpha}\right.$ in $A / \alpha$ in a finite group $G$ whose order is invertible $\}$. We denote the set $\left\{a\right.$ in $A / \alpha^{\prime}(a)=a$ for each $\alpha^{\prime}$ in $\left.G^{\prime}\right\}$ by $A^{G^{\prime}}$ and the projective group ring over $A^{G^{\prime}}$ by $A^{G^{\prime}} G_{f}$ where $f(\alpha, \beta)=U_{\alpha} U_{\beta} U_{\alpha \beta}^{-1}$ for $\alpha, \beta$ in $G$. We shall generalize the characterization of a central Galois algebra as given by F.R. DeMeyer to a non-commutative case. We begin with some properties of G'.

LEMMA 3.1. (1) Let $\sum_{\alpha} C_{\alpha}$ (or $\Sigma_{\alpha} A^{G}{ }^{\prime} U_{\alpha}$ ) be the subring generated by $C$ (or $A^{\prime \prime}$ ) and $\left\{U_{\alpha}\right\}$ respectively. Then G' restricted to $\Sigma_{\alpha} C_{\alpha}$ is isomorphic with G' restricted to $\Sigma_{\alpha} A^{G} U_{\alpha}$. (2) If $\sum_{\alpha} A^{G}{ }^{\prime} U_{\alpha}$ has certer $C$, then $G^{\prime}$ restricted to $\sum_{\alpha} A^{G}{ }^{\prime} U_{\alpha}$ is isomorphic with $G^{\prime}$.

PROOF. Since $\alpha^{\prime}=\beta^{\prime}$ on $\sum_{\alpha} A^{G} U_{\alpha}$ if and only if $U_{\beta}^{-1} U_{\alpha}$ is in the center of $\Sigma_{\alpha} A^{G} U_{\alpha}$, and since $\alpha^{\prime}=\beta^{\prime}$ on $A$ if and only if $U_{\beta}^{-1} U_{\alpha}$ is in $C$ similarly, part (2) holds. Part (1) is clear. LEMMA 3.2. If $A$ is Galois over $A^{G^{\prime}}$ with Galois group $G^{\prime}$, then $G^{\prime} \cong G$.

PROOF. We first show that $\left\{U_{\alpha} / \alpha\right.$ in $\left.G\right\}$ are free cver $C$. In fact, let $\Sigma_{\alpha} a_{\alpha} U_{\alpha}=0$ for $a_{\alpha}$ in $C$ and let $\left\{x_{i}, y_{i}\right.$ in $A, i=1, \ldots, m$ for some integer $m\}$ be a Galois set for $A$. Then $\sum x_{i}\left(\sum_{\alpha} a_{\alpha} U_{\alpha}\right) \beta^{-1}\left(y_{i}\right)$ 
$=0=\sum_{\alpha} a_{\alpha}\left(x_{i} \alpha \beta^{-1}\left(y_{i}\right)\right) U_{\alpha}=a_{\beta} U_{\beta}$ for ary $\beta$ in $G_{0}$ Next, let $\alpha^{\prime}, \beta^{\prime}$ be in $G^{\prime}$ such that $\alpha^{\prime}(a)=\beta^{\prime}(a)$ for all a in $A$; Then $U_{\beta}^{-1} U_{\alpha}$ is in $C$. Thus there is an $c$ in $C$ such that $U_{\beta}^{-1} U_{\alpha}=c$; and so $U_{\alpha}=\mathrm{cU}_{\beta}$. But $\left\{U_{\alpha}\right\}$ are free over $C$, so $U_{\alpha}=U_{\beta}$. Thus $\alpha=\beta$. Moreover, the map $\alpha \rightarrow \alpha^{\prime}$ from $G^{\prime}$ to $G$ is clearly a group homomorphism, so $G^{\prime} \cong$ G.

THEOREM 3.3. Let $A$ be a Galois extension over $A^{G '}$ with Galois group $G^{\prime}$. If $\Sigma_{\alpha} A^{G^{\prime}} U_{\alpha}$ has center $C$, then $A=\sum_{\alpha} A^{G^{\prime}} U_{\alpha}$ which is generated by $A^{G^{\prime}}$ and $\left\{U_{\alpha}\right\}$.

PROOF. As Eiven in the proof of Lemma 3.2, $\left\{U_{\alpha}\right\}$ are free over $C$, so there exists a projective group subalgebra $C_{f}$ of $A$ where $f(\alpha, \beta)=U_{\alpha} U_{\beta} U_{\alpha \beta}^{-1}$ for $\alpha, \beta$ in $G$. Since the order of $G$ is invertible, $(1 / n) \Sigma_{\alpha} U_{\alpha} \otimes U_{\alpha}^{-1}$ is a separable element for $A$ where $n$ is the order of $G$. Hence $C_{f}$ is a sexarable projective group algebra over $C$. Now, let $r$ be an element in the center of $C_{f}$. Then $r U_{\alpha}=U_{\alpha} r$ for each $\alpha$ in $G$. On the other hand, for any $t$ in $A^{G^{\prime}}, U_{\alpha} t U_{\alpha}^{-1}=t$ for each $\alpha$ in $G$, so $U_{\alpha} t=t U_{\alpha}$. Hence $r t=t r$ (for $r$ is in $C G_{f}$ ). Since $C_{f} \subset \Sigma_{\alpha} A^{G} U_{\alpha}, r$ is in the center of $\Sigma_{\alpha} A^{\prime} U_{\alpha}$. By hypothesis, $\Sigma_{\alpha} A^{G} U_{\alpha}$ has center $C$, so $r$ is in $C$. Thus the center of $C G_{f}$ is contained in C. Clearly, $C$ is contained in the center of $C G_{f}$, so $C G_{f}$ has center $C$. Therefore, $C J_{f}$ is an Azumaya algebra over $C$. But then $C_{f}$ is a central Galcis algebra over $C$ with Galois group $G$ ' restricted to $C G_{f}$ by Theorem 3 in [1]. Since $C G_{f} \subset \sum_{\alpha} A^{G} U_{\alpha}$ such that G' restricted to $\Sigma_{\alpha} A^{G} U_{\alpha}$ is isomorphic with $G^{\prime}$ restricted to $C_{f}$ by Lemma 3.1, $\sum_{\alpha} A^{G} U_{\alpha}$ is Galois over $A^{\prime}$ ' with Galois group $G$ ' restricted to $\sum_{\alpha} A^{G '} U_{\alpha}$ (for the Galois set for $C_{f_{f}}$ is also a Galois set for $\Sigma_{\alpha} A^{G}\left(U_{\alpha}\right)$. Moreover, let $\left\{x_{i}, y_{i}\right.$ in $\left.\sum_{\alpha} A^{\prime} U_{\alpha} / i=1, \ldots, k\right\}$ be a Galois

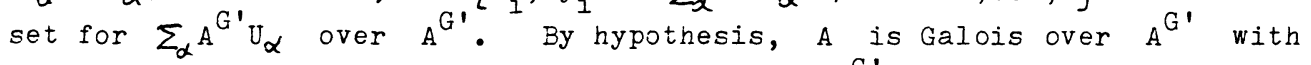
Galois group $G^{\prime}$, so $G^{\prime} \cong G^{\prime}$ restricted to $\sum_{\alpha} A^{\prime} U_{\alpha}$ by Lemma 3.2 . Thus $\left\{x_{i}, y_{i}\right\}$ is also a Galois set for $A$ over $A^{G}$. Tnerefore, $A$ is finitely generated and projective over $A^{G^{\prime}}$ with a dual basis $\left\{\mathrm{x}_{i},\left(\mathrm{tr}_{\mathrm{r}}\right) \mathrm{y}_{i-}\right\}$ where $t_{r}=\Sigma_{\alpha} \alpha$ (the trace of G) ([2], see the proof of Theorem 1, P. 119) Hence, for any a in $A, a=\sum x_{i} \operatorname{tr}\left(y_{i} a\right)$ which is in $\Sigma_{\alpha} A^{\prime} U_{\alpha}$. Thus $A=\Sigma_{\alpha} A^{G} U_{\alpha}$.

We recall that a Galois extension $T$ over $T^{G}$ with Galois group $G$ is called a centralized Galois extension if $A^{G}$ has the same center as $A$. COROLLARY 3.4. By keeping the hypotheses of Theorem 3.3, A is a centralized Galois extension.

PROOF. Clearly, $C$ is contained in the center of $A^{G^{\prime}}$. Conversely, for any $r$ in the center of $A^{G}, r$ is in the center of $\sum_{\alpha} A^{\prime} U_{\alpha}$. By Theorem 3.3, $A=\Sigma_{d} A^{G^{\prime}} U_{\alpha}$, so $r$ is in $C$. Thus the center of $A^{G}$ is also C.

Next is a condition under which $A$ is a projective group ring. THEOREM 3.5. By keeping the hypotheses and notations of Theorem 3.3, if $\Sigma_{\alpha} A^{G} U_{\alpha}$ is separable over $C$, then $A=A^{G} G_{f}$ such that $A{ }^{\prime}$ is an $A z u-$ 
maya C-algebra.

PRCOF. Ey Theorem 3.3, $A=\sum_{\alpha} A^{G '} U_{\alpha}$ containing an Azumaya algebra $C_{f}$ over $C$. By hypothesis, $A$ is an Azumaya C-aleebra containing an Azumaya subalgebra $\tau G_{f}$, so $A \cong Z_{A}\left(C G_{f}\right) \otimes_{C} C G_{f}$ where $Z_{A}\left(C G_{f}\right)$ is the commutant of $C G_{f}$ in $A$ such that $Z_{A}\left(C G_{f}\right)$ is an Azumaya C-subalgebra by the commutant theorem for Azumaya algebras ([3], Theorem 4.3, P. 57). Noting that $Z_{A}\left(C G_{f}\right)=A^{G}$, we conclude that $A \cong A^{G} \otimes_{C} C G_{f} \cong A^{G} G_{f}$ such that $A^{G}$ is an Azumaya'c-algebra.

The following is a property of a Galois projective group ring. THEOREM 3.6. Let $R$ be a ring with 1 (rot necessarily commutative). If $R G_{f}$ is Galois over $\left(R G_{f}\right)^{G^{\prime}}$ with Galois group $G^{\prime}$, then $\left(R G_{f}\right)^{\prime}=R^{\prime}$ FROOF. Let $C$ be the center of $R G_{f}$. Then $C \subset\left(R G_{f}\right)^{G^{\prime}} \cdot$. Since $R G_{f}$ is Galois, $\left\{U_{\alpha}\right\}$ are free over $C$ by the proof of Lemma 3.2 . Hence there exists a projective group subalgebra $C_{f}$ of $R_{f}$. Next, let $D$ be the center of $R$. We claim that $C=D$. In fact, for any $x=\sum_{\alpha} r_{\alpha} U_{\alpha}$ in $C$ and $t$ in $R$, $x t=t x$, so $r_{\alpha}$ are in the center of $R$. But then $C \subset D G_{f}$ Hence $C_{f} \subset D G_{f}$ since $\left\{U_{\alpha}\right\}$ are free over $C$ and $D$ respectively, $C \subset D$. Clearly, $D \subset C$, so $C=D$. Thus the center of $C G_{f}$ is $C$. Noting that the order of $G$ is invertible we conclude that $C_{f}$ is an Azumaya C-algebra; and so $\mathrm{CG}_{f}$ is a central Galois C-algebra with Galois group $G^{\prime}$ ([1], Theorem 3). Moreover, since $R G_{f} \cong R_{C} C G_{f}$ such that $C$ is a $C$-direct summand of $C G_{f}$, there exists an element $d$ in $C G_{f}$ such that $\operatorname{tr}(d)=1$ by using the fact that Hom ${ }_{A^{\prime}}\left(A, A^{\prime}\right) \cong(\operatorname{tr}) A$ (see [2], P. 119 and the introduction to section 2 in [5]). Then, for any $\sum_{\alpha} r_{\alpha} \otimes U_{\alpha}$ in $\left(R G_{f}\right)^{\prime}$ and $\beta$ in $G, \beta\left(\sum_{\alpha} r_{\alpha} \otimes U_{\alpha}\right)=\sum_{\alpha} r_{\alpha}\left(U_{\alpha}\right)=$ $\sum_{\alpha} r_{\alpha} \beta\left(U_{\alpha}\right) \operatorname{tr}(d)=\sum_{\alpha} r_{\alpha} \operatorname{tr}\left(U_{\alpha} d\right)$ which is in $R$ (for $\operatorname{tr}\left(U_{\alpha} d\right)$ is in $C$ ). Thus $\left(R G_{f}\right)^{\prime}=R$.

We now generalize the theorem of $F \cdot R$. DeMeyer that if $K G_{f}$ is Azumaya over a commutative ring $K$, then $K_{f}$ is Galois over $K$ with Galois group G' ([1], Theorem 3).

THEOREM 3.7. If $\Sigma_{\alpha} A^{G} U_{\alpha}$ is an Azumaya C-algebra with $\left\{U_{\alpha}\right\}$ a free set over $C$, then $\Sigma_{\alpha} A^{G}{ }^{\prime} U_{\alpha}=A^{G}{ }^{\prime} G_{f}$ and is a centralized Galois extension over $A^{\prime}$ with Galois group G'.

PROOF. Since $\left\{U_{\alpha}\right\}$ are free over $C, \sum_{\alpha} A^{G}{ }^{\prime} U_{\alpha}$ contains a projective group algebra $C_{f}$. Since the order of $G$ is invertible, $C_{f}$ is separable over $c$. Since the center of $C_{f}$ is in the center of $\sum_{\alpha} A^{\prime} U_{\alpha}$, it is equal to $C$. Hence $C_{f}$ is an Azumaya $C$-algebra. Thus $C_{f}$ is a central Galois C-algebra with Galois group G' ( 1$]$, Theorem 3, and Lemma 3.1). Noting that $C G_{f} \subset \Sigma_{\alpha} A^{G^{\prime}} U_{\alpha}$, we conclude that $\sum_{\alpha} A^{G^{\prime}} U_{\alpha}$ is Galois over $A^{G '}$ with Galois group $G^{\prime}$. Moreover, since $\sum_{\alpha} A^{\prime} U_{\alpha}$ is Azumaya over $C$ by hypothesis, $\sum_{\alpha} A^{G^{\prime}} U_{\alpha}=A^{G^{\prime}} G_{f}$ by the commutant theorem for Azumaya algebras as given in the proof of Theorem 3.5. Thus the proof is complete.

COROLLARY 3.8. Let $R$ be a ring with 1 and $C$ the center of the pro- 
jective group ring $R G_{f}$ over $k$. If $R G_{f}$ is an Azumaya algebra such that $\left\{\mathrm{U}_{\alpha}\right\}$ are free over $C$, then $\mathrm{RG}_{\mathrm{f}}$ is a centralized Galois extension over $R$ with Galois group G'.

PROOF. Let the center of $R$ be $D$. Then there exists a projective group algebra $\mathrm{DG}_{f}$ in $\mathrm{RG}_{f}$. Clearly, $\mathrm{RG}_{f} \cong \mathrm{F}_{\mathrm{D}} \mathrm{DG} \mathrm{G}_{\mp}$. Since $\left\{U_{\alpha}\right\}$ are free over $C$ by hypothesis, we can show that $C=D$ by a similar proof of Theorem 3.6. Moreover, since $a U_{\alpha}=U_{\alpha}$ a for each a in $R$ and $\alpha$ ir $G$, the center of $D G_{f}$ is contained in $C_{0}$ Clearly, $C$ is contained in the center of $D G_{f}$, so $C=$ the center of $D G_{f}$. Hence $D$ = the center of $D G_{f}$. Thus $D G_{f}$ is a central Galois D-algebra. Therefore $R G_{f}$ is Galois over $R$ by Theorem 3.6 such that the center of $R$ is $C$.

We conclude the pacer with two more properties of a Galois projective group ring $R G_{f}$.

THEOREM 3.9. Let $R_{f}$ be a Galois projective group ring with Galois group G' over a ring $R$ and with center $C$. Then (1) the centralizer of the projective group algebra $C G_{f}$ in $R G_{f}$ is $R$, and (2) the center of $R$ is equal to $C$ (and hence $R G_{f}$ is a centralized Galois extension over R).

PROOF. (1) By Theorem 3.6, $R=\left(R_{f}\right)^{G^{\prime}}$. Since $G^{\prime}$ is an inner automorphism group of $R G_{f}$ induced by $\left\{U_{\alpha}\right\}$, part (1) is immediate.

(2) Let $D$ be the center of $R$. Then it is easy to verify that $D_{f}$ $=$ the centralizer of $R$ in $R G_{f}$. By part (1), $D G_{f}=C G_{f}$. Since $R G_{f}$ is Galois, $\left\{U_{\alpha}\right\}$ are free over $C$ by the proof of Lemma 3.2. Eut then $D=C$.

\section{REFERENCES}

1. DeMeyer, F.R. Galois Theory in Separable Algebras over Commutative Rings, Illinois J. Math. 10 (1966), 287-295.

2. DeMeyer, F.R. Some Notes on the General Galois Theory of Rings, Osaka J. Math. 2 (1965), 117-127.

3. DeMeyer, F.R. and E. Ingraham. Separable Algebras over Commutative Rings, Springer-Verlag, Heidelberg-New York, 1971.

4. Chase, S., D. Harrison and A. Rosenberg. Galois Theory and Galois Cohomology of Commutative Rings, Mem. Amer. Math. Soc. No. 52 (1965).

5. Miyashita, Y. Finite outer Galois Theory of Non-commutative Rings, J. Fac. Sci. Hokkaido Univ. Ser. 1, 19 (1966), 114-134.

6. Szeto, G. A Characterization of a Cyclic Galois Extension of Commutative Rings, J. Pure and Applied Algebra 16 (1980), 315-322.

7. Szeto, G. On Separable Abelian Extensions of Rings, Internat. J. Math. and Math. Sci. 4 (1982), 779-784. 


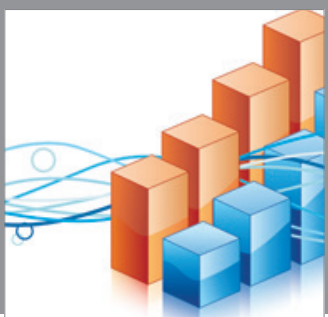

Advances in

Operations Research

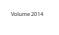

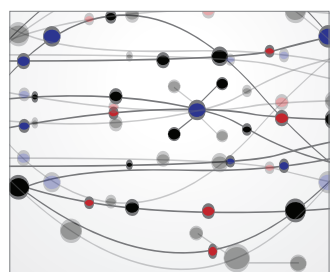

\section{The Scientific} World Journal
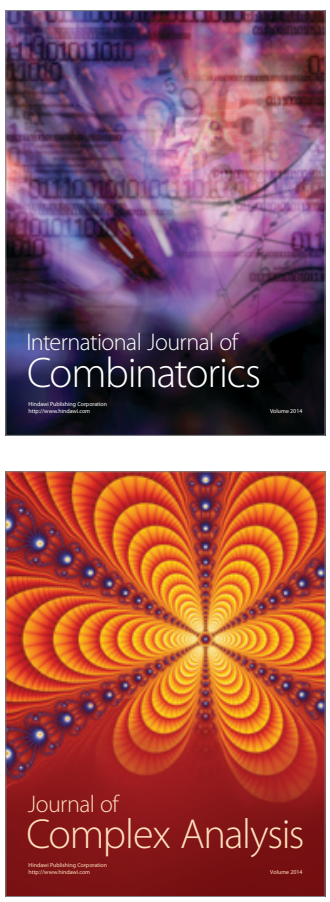

International Journal of

Mathematics and

Mathematical

Sciences
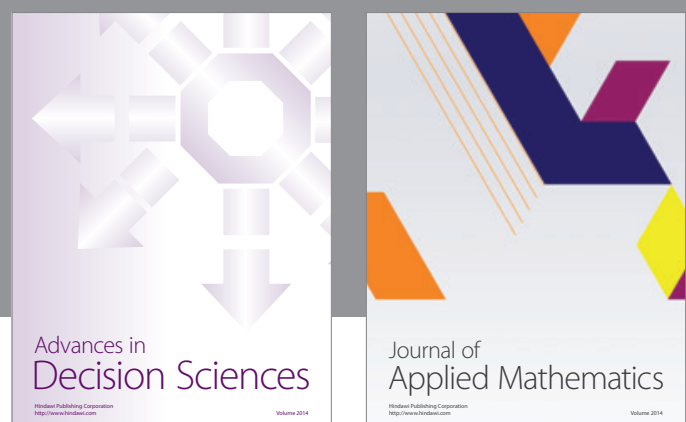

Journal of

Applied Mathematics
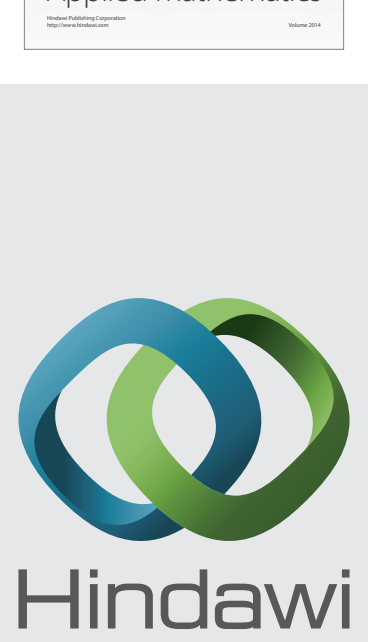

Submit your manuscripts at http://www.hindawi.com
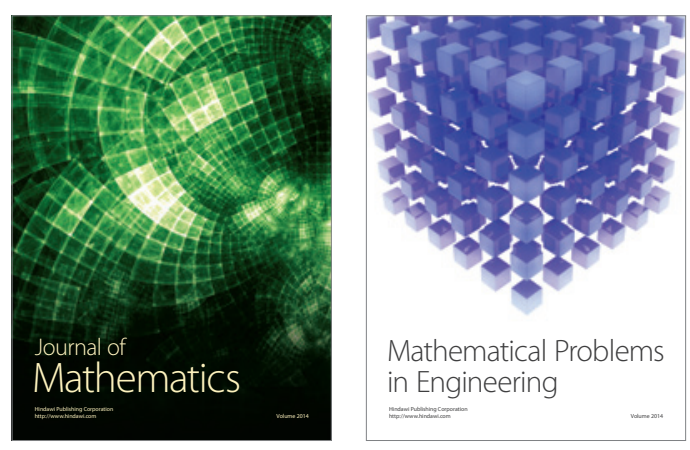

Mathematical Problems in Engineering
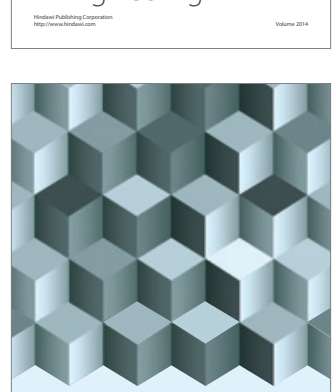

Journal of

Function Spaces
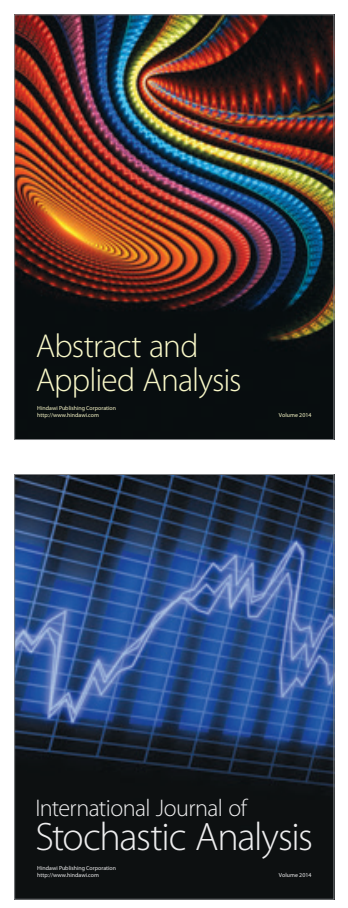

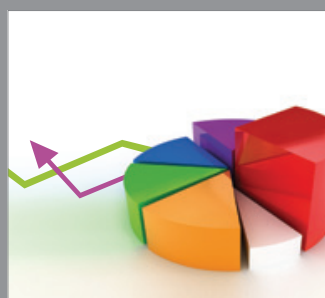

ournal of

Probability and Statistics

Promensencen
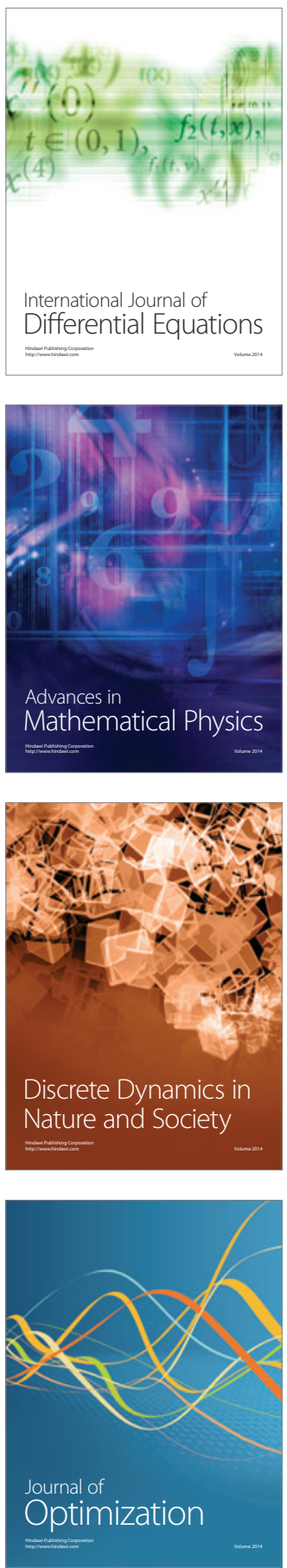